\title{
Positron lifetime studies in vinyl polymers of medical importance
}

\author{
M.F. Ferreira Marques ${ }^{\mathrm{a}, \mathrm{b}, *}$, P.M. Gordo ${ }^{\mathrm{b}}$, C. Lopes Gil ${ }^{\mathrm{b}}$, \\ Zs. Kajcsos ${ }^{\text {b,c }}$, M.H. Gil ${ }^{\text {d }}$, M.J. Mariz ${ }^{\text {d, A.P. de Lima }}{ }^{\text {b' }}$ \\ ${ }^{a}$ Instituto Superior de Engenharia, 3031-199 Coimbra, Portugal \\ ${ }^{\mathrm{b}}$ ICEMS, Departamento de Física, Universidade de Coimbra, 3004-516 Coimbra, Portugal \\ ${ }^{\mathrm{c}}$ KFKI Research Institute for Particle and Nuclear Physics, H-1525 Budapest P.O. Box 49, Hungary \\ ${ }^{\mathrm{d}}$ Departamento de Engenharia Química, Universidade de Coimbra, 3030-290 Coimbra, Portugal
}

\begin{abstract}
Vinyl polymers used as artificial lens implants in ophthalmology were investigated by positron lifetime spectroscopy. The structure of these polymers with free volumes offers the possibility of charging them with anti-inflammatory drugs for sustained release. A correlation between the amount of normalised free volume and the ratio of the methyl methacrylate to ethyl-hexyl-acrylate, used as polymerisation monomers, was found.
\end{abstract}

(C) 2003 Elsevier Science Ltd. All rights reserved.

Keywords: Vinyl polymers; Positronium; Intra-ocular lenses; Pore size; Free volume

\section{Introduction}

The implantation of intra-ocular lenses (IOL) with steadily improving optical properties and tissue-compatibility has been the aim of cataract surgery since its beginnings (Apple, 1992). Anti-inflammatory drugs have to be applied during the operation and also afterwards. Some polyvinyl lenses combine the property of being bendable, allowing small corneal incisions, with a structure containing free volumes, which can be charged by anti-inflammatory drugs, to be slowly released just on the very location where they are needed. Nowadays, with over one million IOLs implanted each year, it is of high interest to contribute to the progress on this field. Knowledge on the size distribution and total fraction of free volumes in the IOLs will be of crucial importance for the control of the containment capability and the release kinetics of anti-inflammatory drugs.

\footnotetext{
*Corresponding author. Instituto Superior de Engenharia, 3031-199 Coimbra, Portugal. Tel.: +351-239-410-600. Fax: +351-239-829-158.

E-mail address: fatima@lipc.fis.uc.pt (M.F. Ferreira Marques).
}

Positron annihilation lifetime spectroscopy (PALS) is a powerful technique for the characterisation of free volume fractions and free volume size distributions (Jean, 1993) in solids. In polymers, the long lifetime component, connected to the ortho-positronium, $o$-Ps, is expected to give information on free volume characteristics.

\section{Experimental}

IOL sample materials were prepared for PALS investigations by the radical polymerisation process using commercial grade chemicals. The monomers were methyl methacrylate (MMA), ethyl-hexyl acrylate (EHA), butyl methacrylate (BMA), and ethyleneglycol dimethacrylate (EGDMA), as cross-linker, from Sigma. The chemical initiator, benzoyl peroxide $(\mathrm{PB})$, was supplied by Aldrich, and the anti-inflammatory drug, flurbiprofen, by Oftalder. The solvents were of analytical grade.

Solutions with different percentages of monomers, cross-linker and initiator were injected into the casting mould and heated in an oven to and kept at $80^{\circ} \mathrm{C}$ for $3 \mathrm{~h}$ 
to accomplish polymerisation. The positron lifetime spectra (LS) were recorded using a fast-fast system, based on $\mathrm{BaF}_{2}$ and Pilot-U scintillators, with a time resolution of about $220 \mathrm{ps}$ FWHM for ${ }^{60} \mathrm{Co}$. The positron source, ca. $7 \times 10^{5} \mathrm{~Bq}$ of ${ }^{22} \mathrm{Na}$, placed between thin nickel foils, was sandwiched between two identical specimens of $1 \mathrm{~cm}^{2}$ area cut from $2 \mathrm{~mm}$ thick polymer sheets. The positron lifetime spectra were recorded slightly above room temperature $(298 \pm 0.5 \mathrm{~K})$ to surpass the glass transition temperature of the polymers. Each sample was counted several times and each lifetime spectrum had ca. $1.7 \times 106$ integral counts. The samples were kept in air, just as during their medical applications.

The spectra were fitted by applying a version of POSITRONFIT (Kirkegaard and Eldrup, 1974), slightly modified for faster convergence. Appropriate source correction was taken into account.

\section{Results and discussion}

The LS spectra were deconvoluted into three components with lifetimes $\tau_{i}$ and intensities $I_{i}$. Subscripts $i=1-3$ are ascribed to $p$-Ps, free $\mathrm{e}^{+}$and $o$-Ps, respectively. The lifetime $\tau_{3}$ and intensity $I_{3}$ are associated with pick-off annihilation of $o$-Ps in free volumes. According to the simple model of Tao and Eldrup (Tao, 1972; Eldrup et al., 1981) for an o-Ps confined in an infinitely deep spherical potential well, there is a correlation between the free-volume hole radius, $R$, and the long-lived lifetime, $\tau_{3}$, in ns

$\tau_{3}=0.5\left[1-\frac{R}{R_{0}}+\frac{1}{2 \pi} \sin \left(\frac{2 \pi R}{R_{0}}\right)\right]^{-1}$,

where $R_{0}=R+\Delta R$, and $\Delta R=1.656 \AA$ (Nakanishi et al., 1987). In terms of the previous free volume model, $I_{3}$ is assumed to be correlated with the concentration of holes and the fractional free volume, measured by $o$-Ps annihilation, can be determined using the relation (Liu et al., 1993):

$\mathrm{FFV}=A V_{\mathrm{f}} I_{3}$,

where $V_{\mathrm{f}}=(4 / 3) \pi R^{3}$ and $A$ is a material dependent normalisation parameter. However, recent studies (Maurer and Schmidt, 2000; Schmidt and Maurer, 2000), in a given amorphous polymer varying the temperature in a broad range, have shown that the intensity $I_{3}$ cannot be well correlated with the amount of free volume whereas, for temperatures above $T_{\mathrm{g}}$ the agreement between the FFV values calculated by Eq. (2) and those obtained by pressure-volume-temperature and equation-of-state (PVT-EOS) analysis was quite acceptable.

In the present study, where polymers with different chemical composition are measured at a constant temperature above $T_{\mathrm{g}}$ and for which appropriate values of the material dependent normalisation parameter are unknown, it is expected that not only $V_{\mathrm{f}}$ but also $I_{3}$ have to be explicitly considered to obtain a reasonable estimate for the variation of total amount of free volume from PALS results. Accordingly, considering that the PALS method should not be regarded as an absolute but rather a relative measurement technique, we adopted the relative free volume value of Eq. (2), normalised to the MMA value, as a measure of the evolution of FFV as the polymer composition changes.

The LS parameters $\left(\tau_{3}\right.$ and $\left.I_{3}\right)$ were obtained for two series of MMA - and BMA - based copolymers with varying EHA content. The radius and free volume fraction results obtained from LS parameters are presented in Fig. 1, together with the drug diffusion coefficient, as determined by kinetic measurements.

The increase of EHA concentration in the MMA samples is observed to increase the dimensions of the cavities present and also to increase of fractional free volume in this copolymer (Fig. 1a and b). The drug diffusion coefficient, Fig. 1c, is also related to the drug transmittance of the polymer matrix.

Fig. 1 shows the results of BMA for the same EHA percentage range as well. The evolutions are quite

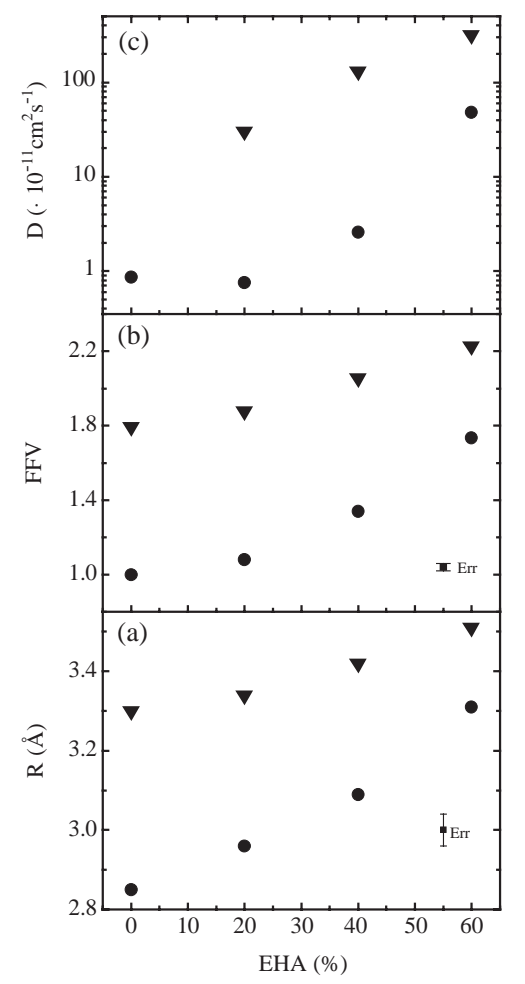

Fig. 1. Variation of free volume radius, $R$ (a), the relative fractional volume, FFV, normalised to the pure MMA monomers (b) and the drug diffusion coefficient (c) for MMA- $(-)$ and BMA-( - based co-polymers with increasing percentage of EHA. 
similar but the values of the radius, the free volume fraction and also the diffusion rates surpass significantly those obtained for MMA. The change from methyl side groups in MMA to butyl groups in BMA may indeed increase the distance between chains. Furthermore, BMA has longer chains than MMA. In both cases the addition of EHA induces the increase of the cavity radius and the free volume fraction values of the copolymer and, consequently, the drug diffusion rate.

Extensive studies have shown that vinyl polymers with $40 \%$ MMA and $60 \%$ EHA, to which $7.5 \%$ in weight of EGDMA cross-linker agent was added, exhibit the best properties for IOL applications (Mariz, 1999). This refers both to transparency, flexibility, drug retainment and drug release rate. For this reason, LS measurements were performed on that basic composition, varying only the amount of cross linker. The results are presented in Fig. 2. For low EGDMA concentrations, the cavity radius and the free volume fraction have no significant changes but the diffusivity decreases strongly reaching the appropriate value for drug release rate at about the selected $7.5 \%$ concentration of EGDMA. For high concentrations, the radius and the free volume fraction are observed to decrease. This may indicate that high

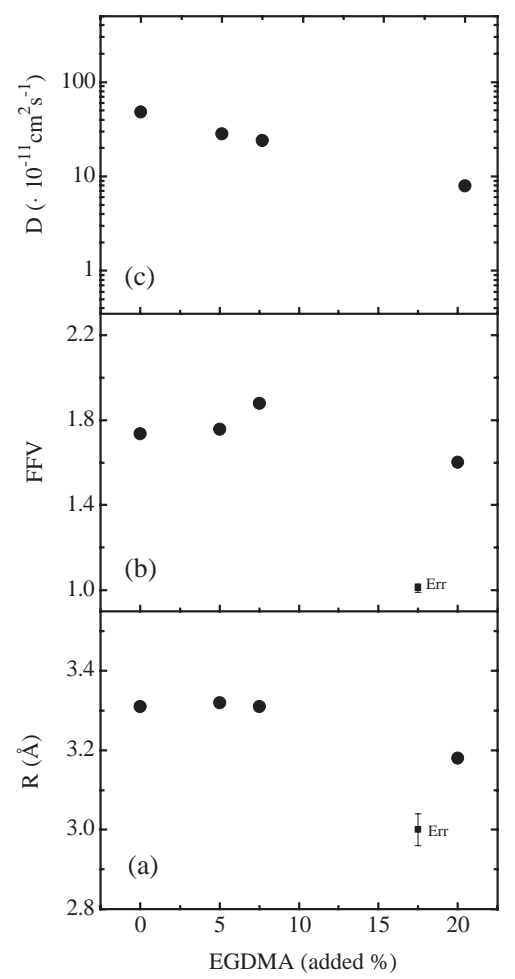

Fig. 2. Variation of free volume radius, $R$ (a), the relative fractional volume, FFV, normalised to the pure MMA monomers (b) and the drug diffusion coefficient (c) for MMA $(40 \%)+$ EHA $(60 \%)$ with different amounts of EGDMA added, percentage in weight. cross-linkage, occupying and reducing the free space, occurs for high EGDMA concentrations. Correspondingly, the drug diffusion coefficient decreases.

The correlation between cavity size and diffusivity, as observed for gas permeation in homogeneous polymers (e.g., Nagel et al., 2002), was not observed here for diffusion of liquids in inhomogeneous polymers.

\section{Conclusions}

Correlations were observed between the radius of the cavities, the effective fractional free volume, the drug release rate and the composition of the vinyl polymers with and without cross-linker. Besides the size of the drug molecules, the drug release rate depends also on the radius of the cavities, the fractional free volume and, naturally, on the interconnectivity of the cavities. The range of free volume values, appropriate for the drug under study, as obtained by LS, was roughly established. These values, characteristic of the polymer, may contribute to optimise the combination of the polymer composition of the IOL with new drugs.

Further work to study the complex questions concerning the correlation between $I_{3}$ and free volume and the dependence of permeability and diffusion constants for these polymers should be undertaken and is in progress.

\section{Acknowledgements}

One of the authors (MFFM) acknowledges the support from Instituto Politécnico de Coimbra. Additional support through the European Commission HPMF-CT-2000-01038/MCFI-2000-01879 contract is highly acknowledged.

\section{References}

Apple, D., 1992. Intraocular lens biocompatibility. J. Cataract Refract. Surg. 18, 217-228.

Eldrup, M., Lightbody, D., Sherwood, J.N., 1981. The temperature dependence of positron lifetimes in solid pivalic acid. Chem. Phys. 63, 51-58.

Jean, Y.C., 1993. Caracterizing free volumes and holes in polymers by positron annihilation spectroscopy. In: Advances with positron spectroscopy of solids and surfaces, NATO, Advanced Research Workshop, pp.563-580.

Kirkegaard, P., Eldrup, M., 1974. Positronfit extended: a new version of a program for analysing positron lifetime spectra. Comput. Phys. Commun. 7, 401-409.

Liu, J., Deng, Q., Jean, Y.C., 1993. Free-volume distributions of polystyrene probed by positron annihilation: comparison with free-volume theories. Macromolecules 26, 7149-7155.

Mariz, M.J., 1999. Preparação de uma lente intra-ocular dotada de um sistema de libertacão controlada de fármaco. 
Mestrado em Ciências da Visão. Faculdade de Medicina da Universidade de Coimbra.

Maurer, F.H.J., Schmidt, M., 2000. Some remarks on the relation between free volume fractions and ortho-positronium lifetimes in amorphous polymers. Radiat. Phys. Chem. $58,509-512$.

Nagel, C., Günther-Schade, K., Fritsch, D., Strunskus, T., Faupel, F., 2002. Free volume and transport properties in highly selective polymer membranes. Macromolecules 35, 2071-2077.
Nakanishi, H., Wang, S.J., Jean, Y.C., 1987. Microscopic surface tension studied by positron annihilation. In: Sharma, S.C. (Ed.), Positron Annihilation Studies of Fluids. World Science, Singapore, pp. 292-298.

Schmidt, M., Maurer, F.H.J., 2000. Relation between free volume quantities from PVT-EOS analysis and PALS. Polymer 41, 8419-8424.

Tao, S.J., 1972. Positronium annihilation in molecular substances. J. Chem. Phys. 56 (11), 5499-5510. 